\title{
Dietary folate protects against the development of macroscopic colonic neoplasia in a dose responsive manner in rats
}

Young-In Kim, R N Salomon, F Graeme-Cook, Sang-Woon Choi, D E Smith, G E Dallal, J B Mason

Vitamin Bioavailability

Laboratory and

Division of

Biostatistics,

Jean Mayer USDA

Human Nutrition

Research Center on

Aging at Tufts

University,

Boston, MA, USA

Young-In Kim

R N Salomon

Sang-Woon Choi

D E Smith

G E Dallal

J B Mason

Divisions of

Gastroenterology and

Clinical Nutrition,

Department of

Medicine,

New England Medical

Center,

Tufts University

School of Medicine,

Boston, MA, USA

Young-In Kim

J B Mason

Department of

Pathology,

New England Medical

Center,

Tufts University

School of Medicine,

Boston, MA, USA

R N Salomon

Department of

Pathology,

Massachusetts General

Hospital,

Harvard Medical

School,

Boston, MA, USA

F Graeme-Cook

Correspondence to:

Dr Young-In Kim,

Department of Medicine,

Room 7258,

Medical Sciences Building,

University of Toronto,

1 King's College Circle,

Toronto, Ontario,

Canada M5S 1 A8.

Accepted for publication

8 July 1996

\begin{abstract}
Background and Aims-Diminished folate status is associated with enhanced colorectal carcinogenesis. This study investigated the potential chemopreventive role of dietary folate in the dimethylhydrazine colorectal cancer model.
\end{abstract}

Subjects and Methods-Sprague-Dawley rats were fed diets containing either 0,2 (daily dietary requirement), 8 or $40 \mathrm{mg}$ folate/kg diet for 20 weeks. After five weeks of diet, rats were injected with dimethylhydrazine (44 mg/kg) weekly for 15 weeks. Fifteen weeks after the first injection of dimethylhydrazine, all rats were killed. Folate status was determined, and the entire colorectum from each rat was analysed for macroscopic and microscopic neoplasms.

Results-Plasma and colonic folate concentrations correlated directly with dietary folate levels $(p<0 \cdot 005)$. The incidence of microscopic neoplasms was similar among the four groups. However, the incidence and the average number of macroscopic tumours per rat decreased progressively with increasing dietary folate levels up to $8 \mathrm{mg} / \mathrm{kg}$ diet $(p<0 \cdot 05)$. In the strongly procarcinogenic milieu used in this study, folate supplementation at 20 times the basal requirement was associated with rates of macroscopic tumour development that were intermediate, and not statistically distinct, from rates observed at either 0 or $8 \mathrm{mg} / \mathrm{kg}$ diet.

Conclusions-These data indicate that in this rat model, (a) increasing dietary folate up to four times the basal requirement leads to a progressive reduction in the evolution of macroscopic neoplasms from microscopic foci; and (b) folate supplementation beyond four times the requirement does not convey further benefit.

(Gut 1996; 39: 732-740)

Keywords: colorectal cancer, chemoprevention, folate, dimethylhydrazine, DNA methylation.

Although genetic alterations have been shown to play an important part in colorectal carcinogenesis, ${ }^{1}$ it has been estimated that up to $90 \%$ of colorectal cancer in the United States can be attributed to dietary factors. ${ }^{2-4}$ Much effort, therefore, has been directed towards defining the relation between nutritional factors and the development of colorectal cancer, and towards the prevention of colorectal cancer through dietary modifications. ${ }^{56}$

Recently, considerable interest has been focused on folate because diminished status of the vitamin has been shown in epidemiological and animal studies to be associated with an increased risk of neoplastic transformation. ${ }^{78}$ Epidemiological studies conducted in people with ulcerative colitis, ${ }^{910}$ as well as in the general population, ${ }^{11-17}$ indicate a $30-50 \%$ reduction in colorectal neoplasia among those with higher, rather than lower, folate status. The most convincing evidence that diminished dietary folate intake is associated with an increased risk of colorectal neoplasia has been recently published by Giovannucci et al. ${ }^{17}$ This prospective study included over 25000 cohorts and correct for the most of the well known dietary confounders associated with colorectal cancer. The study observed a $40 \%$ reduction in the risk of developing colorectal adenomas in subjects with the highest amount of dietary folate intake compared with those with the lowest intake. More recently, prospectively conducted clinical studies have shown that red blood cell ${ }^{18}$ or colonic mucosal ${ }^{19}$ folate concentrations are significantly lower in those harbouring adenomas compared with control subjects, thereby supporting epidemiological findings.

In support of the causal inferences suggested by epidemiological studies, previous work in the dimethylhydrazine (DMH) rodent model of colorectal cancer has shown that moderately folate deplete rats have a significantly greater incidence of microscopic dysplasia and invasive cancer than folate replete controls. ${ }^{20}$ The DMH rodent model of colorectal carcinogenesis mimics the histopathology and molecular biology of the human disease in many respects, ${ }^{21}$ and it has been extensively used in studies that have examined the modulatory effect of nutritional factors on the development of colorectal cancer. ${ }^{21} 22$

To date, the exact mechanisms by which diminished folate status increases colorectal carcinogenesis have not been clearly elucidated, although several candidates have been proposed. ${ }^{8}$ Folate is an essential factor for a number of critical metabolic pathways in the cell that involve the transfer of one carbon groups. Among such pathways are the synthesis of S-adenosylmethionine (SAM), the methyl group donor for most biological transmethylation reactions, including that of 
DNA. ${ }^{23}$ It is in this manner that folate may have an effect on DNA methylation at cytosineguanine dinucleotides ( $\mathrm{CpG}$ ), an epigenetic modification of DNA that is observed in several human and experimental cancers. ${ }^{24}$ Genome wide and certain protooncogene specific DNA hypomethylation has been observed in early stages of colorectal carcinogenesis. ${ }^{1}$ Although studies examining the effect of folate deficiency on genomic DNA methylation remains an unsettled issue, ${ }^{25}{ }^{26}$ folate supplementation at 50 times the daily requirement for humans has recently been shown to be capable of increasing the extent of genomic DNA methylation of the colonic mucosa in subjects with colorectal adenocarcinoma or adenoma. ${ }^{27}$

Previous work in this animal model has merely established that rats moderately deficient in folate develop microscopic neoplasms at a greater rate than rats receiving modest supplementation with the vitamin. ${ }^{20}$ The objectives of this study were to find out if the process by which macroscopic neoplasms evolve from microscopic foci is also suppressed by folate supplementation and to define the dose response nature of this relation. In addition, we investigated the effect of folate deficiency and supplementation, in conjunction with $\mathrm{DMH}$ administration, on colonic mucosal SAM concentrations and genomic DNA methylation because such changes may play a mechanistic part in mediating the enhancement of colorectal carcinogenesis by folate insufficiency.

\section{Methods}

This study was approved by the institutional Animal Care and Use Committee of the Jean Mayer USDA Human Nutrition Research Center on Aging at Tufts University. Forty weanling male Sprague-Dawley rats (60-90 g, Charles River Co, Wilmington, MA) were randomly assigned to receive an amino acid defined diet (Dyets, Bethlehem, PA) ${ }^{28}$ containing either $0,2,8$ or $40 \mathrm{mg}$ folate $/ \mathrm{kg}$ diet $(n=10$, each group). Succinylsulphathiazole, which is conventionally used to create a severe folate deficiency, ${ }^{29}$ was not incorporated into the diets in this study for several reasons. Firstly, we wish to avoid severe folate deficiency, which predictably causes severe growth retardation and premature death ${ }^{29}$; secondly, the consensus of epidemiological and clinical studies $^{9-19}$ and our previous animal study ${ }^{20}$ indicate that mild to moderate depletion of folate is sufficient to increase colorectal carcinogenesis; and thirdly, the use of antibiotics interferes with $\mathrm{DMH}$ activation. ${ }^{30}$ The amino acid defined diets in this study were identical to ones used in a previous study in which rats receiving $0 \mathrm{mg}$ folate $/ \mathrm{kg}$ diet demonstrated a greater incidence of microscopic foci of colonic neoplasia compared with those receiving $8 \mathrm{mg}$ folate $/ \mathrm{kg}$ diet. ${ }^{20}$ Two $\mathrm{mg}$ folate/kg diet is generally accepted as the basal dietary requirement for the rat. ${ }^{31}$ Forty $\mathrm{mg}$ folate/kg diet (that is, 20 times the basal requirement) was chosen in this study because a recent clinical trial observed that folate supplementation in the amount of 50 times the daily requirement for humans was able to modulate a biomarker of colon cancer in humans. ${ }^{27}$ These diets contained $50 \mathrm{~g}$ of cellulose $/ \mathrm{kg}$ as well as $60 \%$ of calories as carbohydrate, $23 \%$ as fat and $17 \%$ as L-amino acids. Rats were housed individually in wire bottomed stainless steel cages to minimise coprophagy. Diets and water were supplied ad libitum. Body weights were recorded weekly. The average daily food consumption of each group was determined on a predetermined day of each week. Five weeks after diet initiation, rats were injected subcutaneously with $44 \mathrm{mg} \mathrm{DMH} .2 \mathrm{HCl}$ (Sigma, St Louis, MO) per kg body weight weekly for 15 weeks. This dose of $\mathrm{DMH}$ is higher than the conventional dose used in induction of colorectal neoplasia in this species of rat, ${ }^{21} 22$ and was deliberately chosen to create a potent procarcinogenic milieu to examine the effect of folate supplementation on the progression of microscopic neoplastic foci to macroscopic tumours. DMH was freshly dissolved in $0.05 \%$ EDTA and adjusted to $\mathrm{pH} 6.5$ with $\mathrm{NaHCO}_{3}$ as described. ${ }^{20}$ All rats were killed 15 weeks after the first injection of DMH (that is, after 20 weeks of the defined diets) by exsanguination under carbon dioxide anaesthesia.

At the time of death, blood was withdrawn from the inferior vena cava using a preheparinised 18 gauge needle into vacutainer tubes containing EDTA and centrifuged at $800 \times g$ for 10 minutes at $4^{\circ} \mathrm{C}$. Plasma was stored at $-70^{\circ} \mathrm{C}$ in $0.5 \%$ ascorbic acid for the plasma folate assay. One hundred microlitre aliquots of plasma were stored without ascorbate for homocysteine assays. Blood samples for complete blood counts were collected into tubes containing sodium EDTA and analysed immediately (System 9000; Serono Baker Diagnostic, Allentown, PA).

The entire colorectum from the caecum to the anus was excised and put on a glass plate suspended on crushed ice. It was then opened longitudinally, rinsed in $0.9 \% \mathrm{NaCl}$, and examined in a blinded fashion for any macroscopic lesions, as defined as any masses or nodules with vascular abnormalities, under a dissecting microscope. All such lesions were mapped and their largest diameter was measured and recorded. Any submucosal thickening without vascular abnormalities (lymphoid follicles) were also measured and mapped. The colorectum was then sectioned longitudinally into two halves of equal width. The mucosal layer from one section was carefully removed by scraping with glass slides. One half of the resulting mucosal scrapings was rapidly weighed, frozen in liquid nitrogen, and stored at $-70^{\circ} \mathrm{C}$ for subsequent analysis of colonic mucosal folate concentrations and DNA extraction. The other half of the mucosal scrapings was immediately homogenised in two volumes of $0.4 \mathrm{M}$ perchloric acid and centrifuged at $1500 \times g$ for 10 minutes, and then the resulting supernatant was frozen at $-70^{\circ} \mathrm{C}$ for subsequent analysis of colonic SAM and $\mathrm{S}$-adenosylhomocysteine (SAH).

The other section was rolled up into a 'Swiss roll', a means by which an intact longitudinal 
section of the entire length of the colorectum can be presented on a single microscope slide, ${ }^{32}$ fixed in $10 \%$ formalin, and processed for light microscopy. A single longitudinal section $(5 \mu \mathrm{m})$ was cut from the midline of each Swiss roll, without regard to the presence of macroscopic lesions, and stained with haematoxylin and eosin. As midline sections were cut without regard to the presence of macroscopic or microscopic lesions, each $5 \mu \mathrm{m}$ section in this study constituted a random and representative longitudinal section from the entire length of the colon of each rat. ${ }^{32}$ This method has previously been used to quantitatively and qualitatively describe $\mathrm{DMH}$ induced lesions in the colorectum. ${ }^{20}{ }^{32}$ Assessment of the microscopic lesions were thereby performed in a manner that was independent of the assessment of macroscopic lesions.

Two experienced pathologists read the slides independently and in a blinded fashion. Two separate readings were obtained to assess concordance, thereby establishing a measure of objectivity in the histopathological analysis. The entire length of each colon was carefully examined for megaloblastic changes and neoplastic foci. Each focus of neoplasia was recorded with respect to its location (proximal $v$ distal) and was classified into low or high grade dysplasia (LGD and HGD, respectively) or invasive adenocarcinoma according to previously described criteria. ${ }^{33}$ The presence of megaloblastosis was determined qualitatively as previously described ${ }^{34}$ : epithelial cells possessing nuclei that were larger and more hyperchromatic than normal and whose chromatin pattern was more 'open' than normal but whose other cytological characteristics, such as columnar shape and basally located nucleus, were retained were considered to be megaloblastic.

Plasma folate concentrations were measured by a microtitre plate assay using Lactobacillus casei. ${ }^{35}$ Colonic mucosal folate concentrations were measured by the same microbiological assay $^{35}$ after extracting in four volumes of fresh folate extraction buffer ( $5 \mathrm{mM} \beta$-mercaptoethano and $0.1 \mathrm{M}$ sodium ascorbate in $0.1 \mathrm{M}$ (bis [2-hydroxyethyl] imino) TRIS [hydroxymethyl]methane, $\mathrm{pH} 7 \cdot 85)^{36}$ followed by treatment with chicken pancreas conjugase to convert all the polyglutamates to their corresponding monoglutamate derivatives. ${ }^{37}$ Total plasma homocysteine was measured by high performance liquid chromatography (HPLC) according to the fluorimetric method of Vester and Rasmussen. ${ }^{38}$ This method incorporates a treatment of the samples with a reducing agent before analysis and, therefore measures the sum of free, protein bound and disulphide forms of homocysteine. Concentrations of colonic mucosal SAM and SAH were determined by HPLC with ultra violet detection. ${ }^{39}$

The DNA from colonic mucosal scrapings were extracted by a standard technique using a lysis buffer containing proteinase $\mathrm{K}$ followed by phenol, chloroform, and isoamyl alcohol organic extraction. ${ }^{40}$ The resulting DNA was precipitated with $1 \mathrm{M} \mathrm{NaCl}$ and $100 \%$ ethanol and was treated with RNAse. The purified
DNA was further dialysed against $10 \mathrm{mM}$ TRIS-HCl, $1 \mathrm{mM}$ EDTA, $\mathrm{pH} 8 \cdot 0$. The size of DNA estimated by agarose gel electrophoresis was $>20 \mathrm{~kb}$ in all instances. No RNA contamination was detected on agarose gel electrophoresis. The final preparations had an A260/280 ratio $>1 \cdot 8$. The purified DNA was stored at $-70^{\circ} \mathrm{C}$ until the DNA methylation assay.

Genome wide methylation status at $\mathrm{CpG}$ from colonic mucosal DNA was determined by the in vitro methyl acceptance capacity of DNA using [ ${ }^{3} \mathrm{H}$-methyl] SAM as a methyl donor and a prokaryotic CpG DNA methyltransferase as previously described and validated. ${ }^{26,40}$ The manner in which this assay is performed produces a reciprocal relation between the endogenous DNA methylation status and the exogenous ${ }^{3} \mathrm{H}$-methyl incorporation. Briefly, two $\mu \mathrm{g}$ of DNA were incubated in $5 \mu \mathrm{Ci}$ of [ ${ }^{3} \mathrm{H}$-methyl] SAM (New England Nuclear Boston, $\mathrm{MA}$; 3-10 Ci/mmol), four units of Sss1 methylase (New England Biolabs, Beverly, MA), one volume Sss 1 buffer $(50 \mathrm{mM} \mathrm{NaCl}, 10 \mathrm{mM}$ TRIS- $\mathrm{HCl}, 10 \mathrm{mM}$ EDTA, $1 \mathrm{mM}$ dithiothreitol, $\mathrm{pH} \mathrm{8.0)}$ in a total volume of $50 \mu \mathrm{l}$ methylation mixture for three hours at $37^{\circ} \mathrm{C}$. Sss 1 was denatured by heating at $65^{\circ} \mathrm{C}$ for 20 minutes. The incubation mixtures were applied onto discs of Whatman DE-81 ion exchange filters (Fisher Scientific Springfield, NJ) using vacuum filtration apparatus, and the discs were then washed with $5 \% \mathrm{NaH}_{2} \mathrm{PO}_{4}$ for 45 minutes. The discs were then dried at $95^{\circ} \mathrm{C}$ for 30 minutes and the resulting radioactivity of the DNA retained in the discs was measured by scintillation counting using a non-aqueous scintillation fluor. The amount of radiolabel bound to a filter from an incubation mixture lacking DNA was used as background and was subtracted from the values obtained with mixtures containing DNA. The background value was always $<1 \%$ of the uptake observed with DNA samples. All analyses were done in duplicate.

For histopathological analyses, the entire population of rats $(n=10)$ from each group were analysed. However, for other biochemical and molecular analyses, adequate amounts of plasma and colonic tissue were not available from one rat from the folate deplete group and hence this rat was excluded from these analyses.

For continuous response variables, differences among dietary groups were assessed by using single factor analysis of variance. When the overall $F$ ratio achieved statistical significance, orthogonal polynomial contrasts, after a square root transformation of dietary folate, and Fisher's least significant differences were used to better understand the nature of the between group differences. For discrete response variables, differences among groups were assessed by using a Kruskal-Wallis nonparametric analysis of variance; the JonkheereTerpstra test was used to test for trend in such instances. For categorical response variables, differences among groups were assessed by Fisher's exact test and Wilcoxon's rank sum test was used to test for trend. Continuous 
response variables were analysed by using SYSTAT, version 5.03 (SPSS Inc, Chicago, IL). Discrete and categorical variables were analysed by using StatXact for SYSTAT, version 1.01 (Cytel Software Corp, Cambridge, MA). All statistical tests were two sided and were considered statistically significant if the observed significance level ( $p$ value) was less than $0 \cdot 05$. Results are expressed as mean (SEM).

\section{Results}

Body weight and average daily food consumption Growth curves were similar in the four groups of rats; at no time point did the mean weights differ significantly among these groups. No premature death occurred. This finding indicates that the folate deficiency in the rats that were fed $0 \mathrm{mg}$ folate $/ \mathrm{kg}$ diet was not severe, otherwise growth retardation or premature death would have occurred. ${ }^{29}$ The average daily food consumptions, which were determined on a pre-assigned day of each week were also similar among the four groups (data not shown).

\section{Folate and SAM status}

As Figure 1 shows, the mean plasma and colonic mucosal folate concentrations were different among the four groups $(p<0.001$, ANOVA) and correlated directly with the levels of dietary folate $(p<0.001$ and $p=0.003$, respectively). However, colonic folate concentrations reached a plateau beyond four times the basal requirement of folate. This finding is probably related to the fact that folate

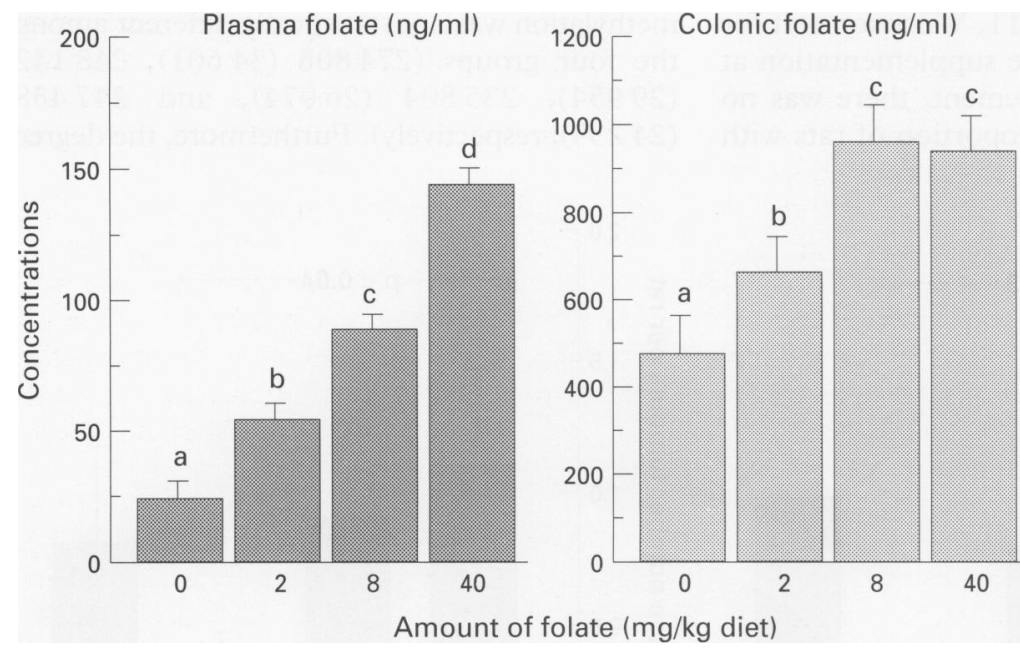

Figure 1: Mean plasma and colonic mucosal folate concentrations in the four groups of rats fed different levels of dietary folate $(n=10$ in each group except in the $0 \mathrm{mg}$ folate/kg group where $n=9)$. At the time of death, blood was collected from the inferior vena cava using a pre-heparinised 18 gauge needle into vacutainer tubes containing EDTA and centrifuged at $800 \times \mathrm{g}$ for 10 minutes at $4^{\circ} \mathrm{C}$, and plasma was stored at $-70^{\circ} \mathrm{C}$ in $0.5 \%$ ascorbic acid for plasma folate assay. The entire colorectum from the caecum to the anus was excised and put on a glass plate suspended on crushed ice. It was then opened longitudinally, rinsed in $0.9 \%$ $\mathrm{NaCl}$, and then sectioned longitudinally into two halves of equal width. The mucosal layer from one section was carefully removed by scraping with glass slides. One half of the resulting mucosal scrapings was rapidly weighed, frozen in liquid nitrogen, and stored at $-70^{\circ} \mathrm{C}$ for subsequent analysis of colonic mucosal folate concentrations. Plasma and colonic mucosal folate concentrations were measured by a microtitre plate assay using

Lactobacillus case ${ }^{38}$ as described in Methods. Both plasma and colonic mucosal folate concentrations correlated directly with the levels of dietary folate $(p<0.001$ and $p=0.003$, respectively). Bars denoted by different letters indicate statistically significant differences by Fisher's least significant difference multiple comparison test $(p<0 \cdot 03)$. accumulation in tissues is limited by the level of folylpolyglutamate synthetase activity in the setting of substrate excess ${ }^{41}$ and is consistent with previous studies in animals and in cultured cells. ${ }^{41-43}$ A highly significant correlation between plasma and colonic mucosal folate concentrations was observed $(r=0.54, \mathrm{p}<0.001)$.

Plasma concentrations of homocysteine, which are known to rise in the setting of folate deficiency, ${ }^{44}$ were increased by $70 \%$ in the folate depleted rats compared with the mean value of the three groups receiving dietary folate $(7 \cdot 26(0 \cdot 70) \mathrm{nmol} / \mathrm{ml} v 4.36(0.32), \mathrm{p}=0.003)$. The mean plasma concentrations among the three groups receiving dietary folate were similar $(4.44(0.63) \mathrm{nmol} / \mathrm{ml}, 4.30(0.59)$, and $4.34(0.47)$, respectively). This is consistent with previous studies that have suggested that folate supplementation at four times the dietary requirement does not lower plasma homocysteine concentrations beyond the level achieved by dietary folate at the daily requirement. ${ }^{39}$ Individual plasma homocysteine values were inversely correlated with the levels of dietary folate $(r=-0.27, \mathrm{p}=0.04)$, plasma folate concentrations $(r=-0.39, \mathrm{p}=0.013)$, and colonic mucosal folate concentrations $(r=-0.36$, $\mathrm{p}=0.025$ ).

Haemoglobin, packed cell value, and mean corpuscular volume were not significantly different among the four groups (data not shown).

Colonic mucosal concentrations of SAM as well as $\mathrm{SAH}$, the second of which accumulates as a result of folate deficiency and which indicates a decreased ability to remethylate homocysteine, ${ }^{23}$ were not significantly different among the four groups (data not shown). Furthermore, the ratio of SAM to SAH, considered by some as a better indicator of methylating capacity, ${ }^{45}$ was similar among the four groups (data not shown).

\section{Microscopic lesion analysis}

The two independent analyses of microscopic lesions by two pathologists were highly correlated: LGD, $r=0.3, \mathrm{p}=0.1 ; \mathrm{HGD}, r=0.6$, $\mathrm{p}<0.001$; and invasive cancer, $r=0.9, \mathrm{p}<0.001$. Furthermore, all analyses pertaining to microscopic data had identical outcomes regardless of which histopathological data set were used (data not shown). Having thereby demonstrated a high degree of concordance, the readings from one of the pathologists were used and reported for all subsequent analyses reported in this paper. Megaloblastic changes in the colonic epithelium were observed in $80 \%, 60 \%, 70 \%$, and $40 \%$ of the rats receiving $0,2,8$, and $40 \mathrm{mg}$ folate/kg diet, respectively. This is consistent with earlier reports that $\mathrm{DMH}$, without the superimposition of folate deficiency, may produce changes simulating megaloblastosis. ${ }^{20}$ The cancers were adenocarcinomas in all instances.

The proportion of rats with LGD, HGD, invasive cancer or any combination thereof was similar among the four groups (Table). Similarly, the proportion of rats with multiple microscopic lesions, in various combinations, 
Percentage of rats with microscopic neoplastic foci in four study groups that received four different levels of dietary folate

\begin{tabular}{|c|c|c|c|c|c|}
\hline & \multicolumn{5}{|c|}{ Amount of dietary folate ( $\mathrm{mg} / \mathrm{kg}$ diet) } \\
\hline & $\begin{array}{l}0 \\
(n=10)\end{array}$ & $\begin{array}{l}2 \\
(n=10)\end{array}$ & $\begin{array}{l}8 \\
(n=10)\end{array}$ & $\begin{array}{l}40 \\
(n=10)\end{array}$ & $p$ Value \\
\hline Low grade dysplasia (LGD) & 80 & 90 & 80 & 70 & $0 \cdot 74$ \\
\hline High grade dysplasia (HGD) & 10 & 20 & 50 & 30 & $0 \cdot 22$ \\
\hline Invasive cancer (CA) & 20 & 30 & 10 & 20 & 0.74 \\
\hline LGD or HGD & 80 & 90 & 80 & 70 & $0 \cdot 74$ \\
\hline $\mathrm{LGD}$ or $\mathrm{HGD}$ or CA & 90 & 90 & 80 & 70 & 0.59 \\
\hline
\end{tabular}

was not statistically different among the four groups (data not shown). A similar analysis, which examined the average number of neoplastic foci per animal, also showed no significant differences in the burden of microscopic foci in the four groups (data not shown). No significant correlation was observed between plasma or colonic folate concentrations and the presence or the number of microscopic neoplasms. Colonic SAM concentrations and SAM/SAH ratios were not significantly correlated with the presence or the number of microscopic lesions.

Macroscopic tumour analysis

Most of the macroscopic tumours (90\%) were located in the distal colon. Non-specific submucosal fold thickening without vascular abnormalities were present in a similar proportion of rats in each group and most of these $(91 \%)$ were located in the proximal colon. Two rats had hepatic, and peritoneal and omental metastatic lesions, respectively: both were in the group receiving $0 \mathrm{mg}$ folate $/ \mathrm{kg}$ diet.

As Figure 2 shows, the proportion of rats harbouring macroscopic tumours decreased progressively with increasing levels of dietary folate up to $8 \mathrm{mg}$ folate $/ \mathrm{kg}$ diet $(p=0.029$, Fisher's exact test; $p=0.011$, Wilcoxon test for trend). With dietary folate supplementation at 20 times the daily requirement, there was no further decrease in the proportion of rats with

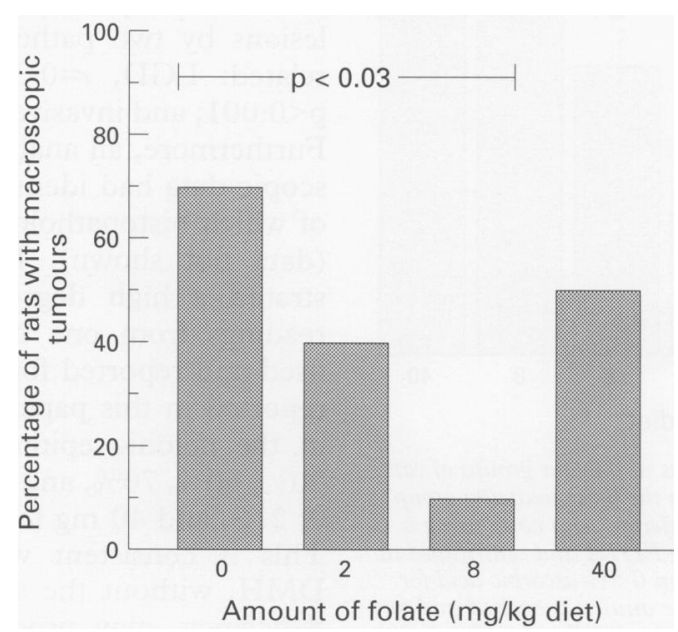

Figure 2: Proportion of rats with macroscopic tumours in the four groups of rats fed different levels of dietary folate $(n=10$ in each group). The proportion of rats with macroscopic tumours decreased progressively with increasing levels of dietary folate up to $8 \mathrm{mg}$ folate $\mathrm{kg}$ diet $(p=0.029$, Fisher's exact test; $p=0 \cdot 011$, Wilcoxon test for trend). Folate 'hypersupplementation' exceeding four times the basal requirement did not convey further inhibition of tumorigenesis. macroscopic tumours compared with the group receiving $8 \mathrm{mg}$ folate $/ \mathrm{kg}$ diet $(\mathrm{p}=0 \cdot 2$, Fisher's exact test). In fact, a non-significant trend towards a larger proportion of rats with macroscopic neoplasms was evident in the $40 \mathrm{mg} / \mathrm{kg}$ group compared with the $8 \mathrm{mg} / \mathrm{kg}$ group.

Similarly, Figure 3 shows that the average number of macroscopic tumours per rat decreased progressively with increasing levels of dietary folate up to $8 \mathrm{mg}$ folate $/ \mathrm{kg}$ diet $(p=0.036$, Kruskal-Wallis; $p=0.016$ Jonkheere Terpstra test for trend) and that a nonsignificant trend towards greater numbers of macroscopic tumours per rat was evident in the group receiving $40 \mathrm{mg}$ folate $/ \mathrm{kg}$ diet compared with those receiving $8 \mathrm{mg}$ folate $/ \mathrm{kg}$ diet.

The size of the macroscopic tumours ranged from $2.0 \mathrm{~mm}$ to $6.7 \mathrm{~mm}$. The average size of the macroscopic tumours were not significantly different among the four groups, although a trend similar to the incidence and the average number of macroscopic tumours per rat was observed $(3.9(0.7) \mathrm{mm}, 3.5(0.1), 2.0$, and $2.9(0.4)$ in the rats receiving $0,2,8$ and $40 \mathrm{mg}$ folate/kg diet, respectively).

Genome wide colonic DNA methylation status

The extent of genome wide colonic DNA methylation was not statistically different among the four groups (274806 (34601), 248142 (29 954), 235804 (26074), and 247438 (24 239), respectively). Furthermore, the degree

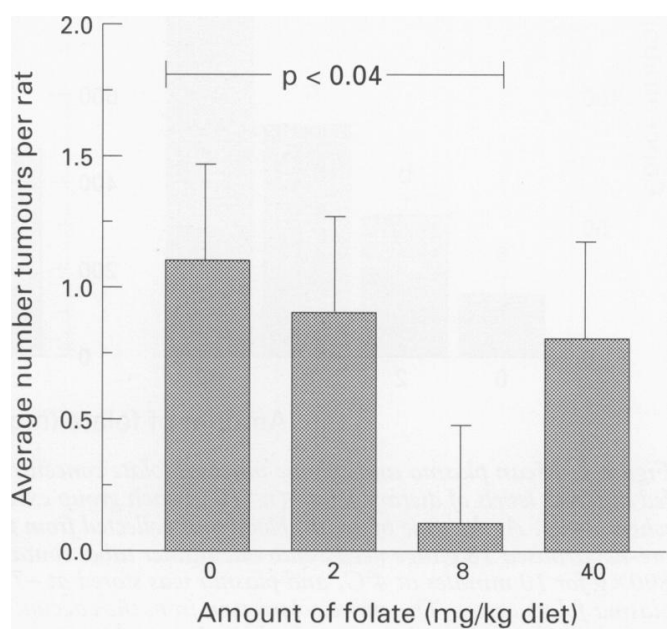

Figure 3: Average number of macroscopic tumours per rat in the four groups of rats receiving different levels of dietary folate ( $n=10$ in each group). The average number of macroscopic tumours per rat decreased progressively with increasing levels of folate up to $8 \mathrm{mg}$ folate $/ \mathrm{kg}$ diet ( $p=0.036$, Kruskal-Wallis, $p=0.016$, Fonkheere-Terpstra test for trend). Folate 'hypersupplementation' exceeding four times the basal requirement did not convey further inhibition of tumorigenesis. 
of genomic methylation was not correlated with either plasma and colonic folate concentrations, colonic SAM concentrations or SAM/SAH ratios or plasma homocysteine levels.

Those rats harbouring macroscopic tumours had a significantly higher mean level of $\left[{ }^{3} \mathrm{H}\right]$ methyl group incorporation into DNA compared with those without (288480 (25 012) $\mathrm{dpm} v 224844$ (14078); $\mathrm{p}=0.03)$, indicating a significantly lower extent of genomic DNA methylation in the rats bearing macroscopic tumours.

\section{Discussion}

This study shows that folate modulates the process of colorectal carcinogenesis over a wide range of dietary intakes in this rodent model of colon cancer, thereby confirming previously published observations from epidemiological, clinical, and animal studies. ${ }^{9-20}$ In particular, these data indicate that a folate deficient diet is associated with a potentiation of the development of macroscopic tumours and that increasing amounts of dietary folate, up to four times the dietary requirement, leads to a progressive reduction in the evolution of macroscopic neoplasms from microscopic neoplastic foci in a dose responsive manner. One interesting finding from this strongly procarcinogenic model is that levels of dietary folate beyond four times the dietary requirement do not convey further benefit. However, because the dose of DMH used in this study was considerably higher than the conventional dose used in induction of colorectal neoplasia, the promoting effect of DMH may have overwhelmed the effect of folate and thus, the magnitude of the folate effect could have been underestimated.

In our previous study, $100 \%$ of the rats that were fed $0 \mathrm{mg}$ folate $/ \mathrm{kg}$ diet developed microscopic neoplasia 20 weeks after receiving $\mathrm{DMH}(20 \mathrm{mg} / \mathrm{kg}$ body weight $)$ injections compared with $29 \%$ of the rats that were fed $8 \mathrm{mg}$ folate $/ \mathrm{kg}$ diet $(\mathrm{p}<0.005) .{ }^{20}$ In contrast, the proportion of macroscopic tumours between the folate depleted and control groups was not significantly different $(86 \%$ v $43 \%, \mathrm{p}=0 \cdot 09) .^{20}$ The earlier study, therefore, suggested that folate deficiency acts on an early phase of $\mathrm{DMH}$ induced colorectal carcinogenesis. ${ }^{20}$ In the present study, the dose of DMH $(44 \mathrm{mg} / \mathrm{kg}$ body weight) was more than twice the dose used in the previous study, and was deliberately chosen to create a very strong procarcinogenic milieu to elucidate more effectively the effect of folate supplementation on the progression of microscopic neoplastic foci to macroscopic tumours. As the induction of colonic neoplasms by $\mathrm{DMH}$ is dose dependent, ${ }^{21}{ }^{22}$ not surprisingly, almost all rats had microscopic neoplastic foci, independent of the levels of dietary folate, at a time point that was five weeks earlier than that of the previous experiment. However, this study clearly demonstrates that folate supplementation renders protection against the evolution of microscopic neoplastic foci to macroscopic tumours in a dose responsive manner. Taken together, these data suggest that folate insufficiency or supplementation has modulatory effects on the development of colorectal neoplasms in rats injected with $\mathrm{DMH}$ and that the exact stages of tumorigenesis on which it has the most profound effect seems to be related to the DMH doses used. The schedule used in this study does not permit us to distinguish whether the modulatory effects of dietary folate are exerted on the initiation or promotional stages of carcinogenesis: future studies are warranted to consider this issue.

The degree of folate depletion observed in the rats fed $0 \mathrm{mg}$ folate $/ \mathrm{kg}$ diet was moderate as evidenced by the plasma and colonic mucosal folate concentrations as well as the absence of growth retardation and premature death. Though the folate depletion was only moderate, there was evidence of biochemically significant folate deficiency, as indicated by the modest rise in plasma homocysteine concentrations compared with those of the folate replete rats. This is consistent with other observations that indicate that this magnitude of folate depletion is known to be associated with functionally significant degree of cellular folate depletion within the colonic mucosa. ${ }^{46}$ Therefore, the finding that even a mild to moderate degree of folate depletion can potentiate the action of $\mathrm{DMH}$ on the development of macroscopic tumours in rats further substantiates the epidemiological and clinical studies that have suggested that a modest reduction in folate status is all that is necessary for enhancement of colorectal carcinogenesis when other risk factors are present. ${ }^{9-20}$ Moreover, the fact that those rats fed the daily requirement of folate had a higher incidence of macroscopic tumours compared with those supplemented with folate at four times the daily requirement is consistent with observations from some of the epidemiological studies where the risk of developing colorectal cancer or its precursor, adenoma, is decreased in subjects taking higher amounts of dietary folate than the recommended daily allowance. ${ }^{17}$

Homocysteine is significantly increased in the folate depleted animals compared with the replete groups even though colonic mucosal SAM, SAH, and SAM/SAH ratio are not significantly changed in this group. Interestingly, colonic SAH is not increased in the folate depleted animals even though homocysteine accumulation favours a backwards reaction whereby SAH is created. These observations are consistent with an earlier study where SAM and SAH from the liver and colonic mucosa were compared in rats receiving 0 and $8 \mathrm{mg}$ folate $/ \mathrm{kg}$ diet. ${ }^{26}$ In that study, plasma homocysteine concentrations were threefold higher in the folate depleted rats compared with the folate replete animals, an effect that was present in association with a significant increase in hepatic SAH and significant decrease in hepatic SAM. However, in the earlier study, ${ }^{26}$ as is true in this one, no significant changes in colonic SAM and SAH were observed. This is probably related to the fact 
that the degree to which one carbon metabolism is affected by folate depletion is highly tissue dependent. ${ }^{36}$ Furthermore, it reflects the fact that homocysteine is distributed in the body in a highly compartmentalised and heterogeneous fashion ${ }^{47}$ and therefore an accumulation of homocysteine in the plasma does not necessarily imply its accumulation in the colonic mucosa.

We observed a highly significant positive correlation between the plasma concentration of folate and the concentration of folate in the colonic mucosa; this is consistent with earlier studies in rats that observed similarly strong correlations between serum folate levels and folate levels in the gastrointestinal tract. ${ }^{48}$ Nevertheless, in a recent study in humans, ${ }^{49}$ no such correlation was observed between either plasma or red blood cell folate concentrations and concentrations in isolated colonocytes. In the instance of this second study, colonocytes were first isolated and allowed to equilibrate for several hours before the determination of folate content. In contrast, our method stabilises the folate content at the time of tissue isolation and is furthermore designed to determine the folate concentration of all the contents of colonic mucosa, including epithelial mucous and cells from the lamina propria, both of which are important constituents of the environment in which colonic epithelial cells exist. Therefore, the apparent discrepancy between our study and the abovementioned human study maybe ascribable to species differences or methodological differences: future studies should help clarify this issue.

The observations in this study indicate that the significant, and progressive, decline in colorectal neoplasia that is observed with increasing levels of dietary folate does not continue at levels of folate that exceed four times the daily requirement. The fact that there is no significant difference in the incidence of macroscopic neoplasia between the 8 and $40 \mathrm{mg} / \mathrm{kg}$ groups (see Figs 2 and 3 ) may merely reflect the fact that maximal mucosal content of folate is achieved on the $8 \mathrm{mg} / \mathrm{kg}$ diet (Fig 1). Nevertheless, a nonsignificant trend towards increased macroscopic neoplasms in the $40 \mathrm{mg} / \mathrm{kg}$ group is evident in Figures 2 and 3 and may indicate a difference in the inhibition of carcinogenesis between the 8 and $40 \mathrm{mg} / \mathrm{kg}$ groups. A definitive explanation for this trend is not available from our observations, but it should be interpreted in light of the potent procarcinogenic milieu that was used in this study. Supplemental folate may have two distinct actions in this setting: at lower levels of supplementation it seems to possess an inhibitory effect on genesis of microscopic foci of neoplasia, as has been previously described, ${ }^{20}$ as well as the evolution of macroscopic neoplasms from microscopic ones. Independently, in a strongly procarcinogenic environment where the appearance of microscopic neoplasms is inevitable, exceptionally high supplemental levels may promote the growth of microscopic neoplasms: an 'acceleration phenomenon', which has previously been described in humans who have well established cancers and who are given exceptionally large doses of folate. ${ }^{50}$ Studies incorporating larger numbers of animals are necessary to fully determine whether the upward trend between 8 and $40 \mathrm{mg} / \mathrm{kg}$ reflects a true difference.

The mechanisms by which folate insufficiency increases the risk of, and folate supplementation conveys protection against, colorectal neoplasia remain unclear at present. Because of the intimate relation of folate to biological methylation reaction $\mathrm{s}^{23}$ and the role of aberrant patterns of DNA methylation in carcinogenesis, ${ }^{24}$ several investigators have proposed that one such mechanism might be altered DNA methylation as a result of change in folate status. ${ }^{7820}$ However, the genomic DNA methylation data from our study indicate that moderate folate depletion, and supplementation up to 20 times the daily requirement, in conjunction with $\mathrm{DMH}$ treatment, were not associated with changes in genomic DNA methylation. These data suggest that the modulatory effect of dietary folate on colonic neoplasia in the DMH model is not mediated by changes in genomic DNA methylation. This is consistent with recent observations that indicate that a moderate degree of folate depletion, equivalent to that of the rats fed $0 \mathrm{mg}$ folate/ $\mathrm{kg}$ diet in the present study, does not induce genome-wide or c-myc protooncogene specific DNA hypomethylation in the colon. ${ }^{26}$

There are several possible explanations why alterations in dietary folate were not observed to alter genomic DNA methylation in the colon. Firstly, the colonic mucosal SAM concentration and the ratio of SAM to SAH, two critical determinants in biological methylation ${ }^{23}{ }^{45}$ were similar among the four groups of rats. This may be due to the fact that modulation of SAM and SAH in some tissues such as the colonic mucosa might be particularly resistant to the effect of folate deficiency or supplementation, as indicated by a previous study. ${ }^{26}$ Furthermore, it is known that $\mathrm{DMH}$ treatment increases colonic SAM and SAM:SAH ratio in rats. ${ }^{51}$ Therefore, in this study, the effect of DMH on colonic SAM and SAH might have hidden the effect conveyed by alterations in folate status. Another possible explanation for the lack of change in genomic DNA methylation status in this study relates to a direct effect of $\mathrm{DMH}$ on the assay utilised to assess DNA methylation. Agents that form adducts at the guanine residue in $\mathrm{CpG}$ dinucleotide sequences, such as $\mathrm{DMH}$, can interfere with the binding of DNA methylase to $\mathrm{CpG}$ sequences. ${ }^{52-55}$ Therefore, $\mathrm{DMH}$ induced adduct formation at the guanine residue in $\mathrm{CpG}$ may render these sites a poor substrate for de novo methylation by the Sss 1 methylase utilised for assay in this study; the effect of either folate depletion or supplementation might have been masked as a result. However, consistent with human data, ${ }^{24} 27$ the rats harbouring macroscopic tumours had a significantly lower degree of genomic DNA methylation compared with those without. 
Regardless of its explanation, the lack of altered genomic DNA methylation implies that other mechanisms are responsible for mediating the modulatory effect of dietary folate. Recently, two other mechanisms by which folate can modulate colorectal carcinogenesis have been proposed. ${ }^{8}$ Folate is an essential factor in the de novo biosynthesis of purines and thymidylate $e^{7,8}$ and it is in this manner that folate plays a key part in DNA replication and cell division. Therefore, folate deficiency could contribute to DNA damage and impaired DNA repair, both of which are considered to be important in carcinogenesis. A deficiency of folate in a cell culture system has been observed to potentiate genomic DNA strand breaks induced by DNA damaging agents. ${ }^{56}$ Sustained folate deficiency has recently been observed to induce DNA strand breaks both at the genomic level and within a highly conserved area (exons 5 through 8 ) of the p53 tumour suppressor gene in rat liver. ${ }^{57}$ Because DNA strand breaks are associated with neoplastic transformation ${ }^{58}$ and the $\mathrm{p} 53$ gene is the most frequently implicated gene in colorectal carcinogenesis, ${ }^{59}$ genomic or gene specific DNA strand breaks, or both, may be a mechanism by which folate insufficiency increases colorectal carcinogenesis. Folate deficiency has also been shown to cause an imbalance in deoxynucleotide pools; reduction in the deoxythymidylate pool as a result of folate deficiency leads to expansion of the deoxyuridylate pool and to misincorporation of uracil into newly synthesised DNA. ${ }^{60}{ }^{61}$ Such misincorporation results in abnormal DNA replication and repair. Furthermore, preliminary experiments indicate that folate deficiency impairs DNA excision repair as well as mismatch repair in the rat colon. ${ }^{62}$ Although these mechanisms have not yet been shown to be operative in the folate mediated modulation of colorectal carcinogenesis in the $\mathrm{DMH}$ rodent model, the aforementioned candidate mechanisms provide biologically sound explanations for a causal relation between diminished folate status and colorectal cancer and for the protective effect of folate supplementation on colorectal carcinogenesis.

The manner in which the colons were processed in this study precluded histological analysis of the macroscopic lesions. Nevertheless, there is a compelling body of evidence in rodent models of colon cancer, utilising either DMH or its metabolites, which indicates that macroscopic lesions invariably contain neoplastic foci. ${ }^{32}{ }^{63-70} \mathrm{~A}$ deliberate choice was made at the outset of this study to include only those macroscopic lesions with features of neoplasia as described in the literature ${ }^{63-70}$ : non-specific submucosal thickening without vascular abnormalities were specifically excluded from the macroscopic tumour analysis. Most of the macroscopic neoplastic lesions were located in the distal colon, consistent with other studies, ${ }^{63-70}$ whereas almost all submucosal thickenings were found in the proximal colon near the caecum.

In summary, these data corroborate earlier observations that raised folate status may play an inhibitory part in DMH induced colorectal carcinogenesis. These data indicate that the progression from microscopic to macroscopic neoplasms is inhibited by dietary folate. These data also indicate that this modulatory effect extends over a wide range of folate status. Nevertheless, at levels of supplementation exceeding four times the basal requirement, no protective effect was apparent. The implications for chemoprevention in humans are provocative, but unclear at the present time.

The authors would like to thank the animal caretakers of the Jean Mayer USDA Human Nutrition Research Center on Aging at Tufts University's Department of Comparative Biology and Medicine for the feeding and maintenance of the rats used in this study. We also thank Marie Nadeau and Becky Norum for technical assistance, and Janice Wallace for processing rat colons for histological analysis.

processing rat colons for histological analysis.
This project has been supported in part by a grant from the This project has been supported in part by a grant from the
National Cancer Institute (1UO1 CA63812-01; JBM), a National Cancer Institute (1UO1 CA63812-01; JBM), a
Research Fellowship from the Medical Research Council of Canada (Y-IK) and the US Department of Agriculture, Agricultural Research Service Contract 53-K06-01 (JBM, RNS, DES, GED). The contents of this publication do not necessarily reflect the view or policies of the US Department of Agriculture, nor does mention of trade names, commercial products, or organisation imply endorsement by the US Goverment.

Presented in part at the 95th Annual Meeting of the American Gastroenterological Association, 14-17 May, 1995, San Diego, CA, USA and published in abstract form in Gastroenterology 1995; 108: A489.

1 Fearon ER, Jones PA. Progressing toward a molecular description of colorectal cancer development. FASEB f 1992; 6: 2783-90.

2 Doll R, Peto R. The causes of cancer: Quantitative estimates of avoidable risks of cancer in the United States today. $¥$ Natl Cancer Inst 1981; 66: 1191-308.

3 Levin B. Nutrition and colorectal cancer. Cancer 1992; 70: 1723-6.

4 Wargovich MJ, Baer AR, Hu PJ, Sumiyoshi H. Dietary factors and colorectal cancer. Gastroenterol Clin North Am 1988; 17: 727-45.

5 Vargas PA, Alberts DS. Primary prevention of colorectal cancer through dietary modification. Cancer 1992; 70: 1229-35.

6 Bruce WR, McKeown-Eyssen G, Ciampi A, Dion PW, Boyd N. Strategies for dietary intervention studies in colon cancer. Cancer 1981; 47: 1121-5.

7 Mason JB. Folate status: effects on carcinogenesis. In: Bailey LB, ed. Folate in health and disease. New York: Marcel Dekker, 1995: 361-78.

8 Mason JB. Folate and colonic carcinogenesis: searching for a mechanistic understanding. $₹$ Nutr Biochem 1994; 5: a mech.

9 Lashner BA, Heidenreich PA, Su GL, Kane SV, Hanauer SB. Effect of folate supplementation on the incidence of dysplasia and cancer in chronic ulcerative colitis. A case-control study. Gastroenterology 1989; 97: 255-9.

10 Lashner BA. Red blood cell folate is associated with the development of dysplasia and cancer in ulcerative colitis. f Cancer Res Clin Oncol 1993; 119: 549-54.

11 Benito E, Stiggelbout A, Bosch FX, Obrador A, Kaldor J, Mulet $\mathrm{M}$, et al. Nutritional factors in colorectal cancer risk: A case-control study in Majorca. Int $\mathcal{F}$ Cancer 1991; risk: A case-c $161-7$.

12 Freudehneim $J L$, Graham S, Marshall JR, Haughey BP, Cholewinski S, Wilkinson G. Folate intake and carcinogenesis of the colon and rectum. Int $\mathcal{f}$ Epidemiol 1991; 20: 368-74.

13 Benito E, Cabeza E, Moreno V, Obrador A, Bosch FX. Diet and colorectal adenomas: A case-control study in Majorca. Int 7 Cancer 1993; 55: 213-9.

14 Meyer F, White E. Alcohol and nutrients in relation to colon cancer in middle-aged adults. Am $\mathcal{F}$ Epidemiol 1993; 138: 225-36.

15 Ferraroni M, La Vecchia C. D'Avanzo B, Negri E, Franceschi S, Decarli A. Selected micronutrient intake and the risk of colorectal cancer. Br $\mathcal{F}$ Cancer 1994; 70: and the $1150-5$.

16 Boutron MC, Senesse P, Faivre J. Folate, alcohol and the adenoma-carcinoma sequence. Gastroenterology 1955; 108: A450.

17 Giovannucci E, Stampfer MJ, Colditz GA, Rimm EB, Trichopoulos D, Rosner BA, et al. Folate, methionine, and alcohol intake and risk of colorectal adenoma. $\mathcal{F}$ Natl Cancer Inst 1993; 85: 875-83.

18 Paspatis GA, Kalafatis E, Oros L, Xourgias V, Koutsioumpa P, Karamanolis DG. Folate status and adenomatous colonic polyps. A colonoscopically controlled study. Dis Colon Rectum 1995; 38: 64-8.

19 Kim YI, Fawaz K, Knox T, Arora S, Lee YM, Paiva L, et al. Lower colonic mucosal folate concentrations in patients with adenomatous polyps compared to those with hyperplastic polyps: possible implications for the development of colorectal cancer. Gastroenterology 1994; 106: A402. 
20 Cravo ML, Mason JB, Dayal Y, Hutchinson $M$, Smith D, Selhub J, et al. Folate deficiency enhances the development of colonic neoplasia in dimethylhydrazine-treated rats. Cancer Res 1992; 52: 5002-6.

21 Rogers AE, Nauss KM. Rodent models for carcinoma of the colon. Dig Dis Sci 1985; 30 (suppl): 87-102S.

22 Autrup H, William GM. Experimental colon carcinogenesis. Boca Raton: CRC Press, 1983.

23 Selhub J, Miller JW. The pathogenesis of homocysteinemia: interruption of the coordinate regulation by $\mathrm{S}$-adenosylmethionine of the remethylation and transsulfuration of homocysteine. Am $\mathcal{F}$ Clin Nutr 1992; 55: 131-8.

24 Laird PW, Jaenisch R. DNA methylation and cancer. Hum Mol Genet 1994; 3: 1487-95.

25 Balaghi M, Wagner C. DNA methylation in folate deficiency: Use of $\mathrm{CpG}$ methylase. Biochem Biophys Res Commun 1993; 193: 1184-90.

26 Kim YI, Christman JK, Fleet JC, Cravo ML, Salomon RN, Smith D, et al. Moderate folate deficiency does not cause global hypomethylation of hepatic and colonic DNA or c-myc specific hypomethylation of colonic DNA in rats. Am $\mathcal{F}$ Clin Nutr 1995; 61: 1083-90.

27 Cravo M, Fidalgo P, Pereira AD, Gouveia-Oliveira A, Chaves P, Selhub J, et al. DNA methylation as an intermediate biomarker of colorectal cancer: modulation by folic acid supplementation. Eur $\mathcal{F}$ Cancer Prev 1994; 3: 473-9.

28 Walzem RL, Clifford AJ. Folate deficiency in rats fed diets containing free amino acids or intact proteins. $f$ Nutr containing free amin

29 Clifford AJ, Wilson DS, Bills ND. Repletion of folatedepleted rats with an amino acid-based diet supplemented with folic acid. F Nutr 1989; 119: 1956-61.

30 Goldin BR, Gorbach SL. Effect of antibiotics on incidence of rat intestinal tumor induced by 1,2-dimethylhydrazin dihydrochloride. $\mathcal{F}$ Natl Cancer Inst 1981; 67: 877-80

31 Reeves PG, Nielsen FH, Fahey Jr GC. AIN-93 purified diets for laboratory rodents: Final report of the American Institute of Nutrition ad hoc writing committee on the reformulation of the AIM-76A rodent diet. $\mathcal{f}$ Nutr 1993 ; 123: 1939-1951.

32 Filipe MI. Mucus secretion in rat colonic mucosa during carcinogenesis induced by dimethylhydrazine. A morphological

33 Riddell RH, Goldman H, Ransohoff DF, Appelman HD, Fenoglio CM, Haggitt RC, et al. Dysplasia in inflammatory bowel disease: standardized classification with provisional clinical applications. Hum Pathol 1983; 14: 931-68.

34 Bianchi A, Chipman D, Dreskin A, Rosenzweig N. Nutritional folic acid deficiency with megaloblastic changes in the small

35 Tamura T. Microbiological assay of folate. In: Picciano MF, Stokstad ELR, Greogory JF, eds. Folic acid metabolism in health and disease. New York: Wiley-Liss, 1990: 121-37.

36 Varela-Moreiras G, Selhub J. Long-term folate deficiency alters folate content and distribution differentially in rat tissues. ₹ Nutr 1992; 122: 986-91.

37 Wilson SD, Horne DW. Use of glycerol-protected Lactobacillus casei for microbiological assay of folic acid. Clin Chem 1982; 28: 1198-200.

38 Vester B, Rasmussen K. High performance liquid chromatography method for rapid and accurate determination of homocysteine in plasma and serum. Eur $¥$ Clin Chem Clin Biochem 1991; 29: 549-54.

39 Miller JW, Nadeau MR, Smith J, Smith D, Selhub J. Folatedeficiency-induced homocysteinemia in rats: disruption of S-adenosylmethionine's co-ordinate regulation of homocysteine metabolism. Biochem $₹$ 1994; 298: 415-9.

$40 \mathrm{Kim}$ YI, Giuliano A, Hatch KD, Schneider A, Nour MA Dallal GE, et al. Global DNA hypomethylation increases progressively in cervical dysplasia and carcinoma. Cancer 1994; 74: 893-9.

41 Shane B. Folate chemistry and metabolism. In: Bailey LB, ed. Folate in health and disease. New York: Marcel Dekker, 1995: 1-22.

42 Lowe KE, Osborne CB, Lin B-F, Kim J-S, Hsu J-C, Shane $B$. Regulation of folate and one carbon metabolism in mammalian cells. II. Effect of folylpolyglutamate synthetase substrate specificity and level of folate metabolism and folylpolyglutamate specificity of metabolic cycles of one carbon metabolism. $\mathcal{F}$ Biol Chem 1993; 268: 21665-73.

43 Houghton JA, Williams LG, de Graaf SS, et al. Relationship between dose rate of [6RS]leucovorin administration, between dose rate of [6RS]leucovorin administration,
plasma concentrations of reduced folates and pools of plasma concentrations of reduced folates and pools of 5,10 -methylenetetrahydrofolates and tetrahydrofolates in human colon aden

44 Stabler SP, Marcell PD, Podell ER, Allen RH, Savage DG, Lindenbaum J. Elevation of total homocysteine in the serum of patients with cobalamin or folate deficiency detected by capillary gas chromatography-mass spectrometry. $\mathcal{F}$ Clin Invest 1988; 81: 466-74.
45 Hoffman DR, Marion DW, Cornatzer WE, Duerre JA. $S$-adenosylmethionine and S-adenosylhomocysteine metabolism in isolated rat liver. F Biol Chem 1980; 255: $10822-7$.

46 Cravo ML, Mason JB, Selhub J, Rosenberg IH. Use of the deoxyuridine suppression test to evaluate localized folate deficiency in rat colonic epithelium. Am f Clin Nutr 1991; 53: $1450-4$.

47 Ueland P, Helland S, Broch O, Schanche J-S. Homocysteine in tissues of the mouse and rat. $\mathcal{F}$ Biol Chem 1984; 259: $2360-4$.

48 Clifford AJ, Heid MK, Muller HG, Bills ND. Tissue distribution and prediction of total body folate of rats. f Nutr 1990; 120: 1633-9.

49 Meenan J, O'Hallinan E, Lynch S, Molloy A, McPartlan J, Scott J, Weir DG. Folate status of gastrointestinal epithelial cells is not predicted by serum and red cell folate values in replete subjects. Gut 1996; 38: 410-3

50 Farber S. Some observations on the effect of folic acid antagonists on acute leukemia and other forms of incurable cancer. Blood 1949; 4: 160-7.

51 Halline AG, Dudeja PK, Brasitus TA. 1,2-dimethylhydrazine-induced premalignant alterations in the $\mathrm{S}$-adenosylmethionine/S-adenosylhomocysteine ratio and membrane lipid lateral diffusion of the rat distal colon. Biochim Biophys Acta 1988; 944: 101-7.

52 Hepburn PA, Margison GP, Tisdale MJ. Enzymatic methylation of cytosine in DNA is prevented by adjacent
$\mathrm{O}^{6}$-methylguanine residues. $\mathcal{f}$ Biol Chem 1991; 266: 7985-7.

53 Bolden A, Ward C, Siedlecki JA, Weissbach A. DNA methylation. Inhibition of de novo and maintenance methylation in vitro by RNA and synthetic polynucleotides. $f$ Biol Chem 1984; 259: $12437-43$.

54 Weitzman SA, Turk PW, Milkowski DH, Kozlowski K. Free radical adducts induce alterations in DNA cytosine methylation. Proc Natl Acad Sci USA 1994; 91: 1261-4.

55 Cox R. DNA methylase inhibition in vitro by N-methylN'-nitro-N-nitrosoguanidine. Cancer Res 1980; 40: 61-3.

56 Branda RF, Blickensderfer DB. Folate deficiency increases genetic damage caused by alkylating agents and
$\gamma$-irradiation in Chinese hamster ovary cells. Cancer Res 1993; 53: 5401-8.

57 Kim YI, Pogribny I, Basnakian AG. Miller JW, Selhub J, James SJ, Mason JB. Folate deficiency in the rat induces DNA strand breaks and hypomethylation within the $\mathrm{p} 53$ tumor suppressor gene. Am f Clin Nutr (in press).

58 Borek C, Ong A, Morgan WF, Cleaver JE. Morphological transformation of $10 \mathrm{~T} 1 / 2$ mouse embryo cells can be initiated by DNA double-strand breaks alone. Mol Carcinog 1991; 4: 243-7.

59 Hollstein M, Sidransky D, Vogelstein B, Harris CC. P53 mutations in human cancers. Science 1991; 253: 49-53.

60 James SJ, Cross DR, Miller BJ. Alterations in nucleotide pools in rats fed diets deficient in choline, methioinine and/or folic acid. Carcinogenesis 1992; 13: 2471-4.

61 Sedwick W, Kutler M, Brown O. Antifolate-induced misincorporation of deoxyuridne monophosphate into DNA. Proc Natl Acad Sci USA 1981; 78: 917-21.

62 Choi SW, Kim YI, Weitzel JN, Mason JB. Impaired DNA repair in the rat colon is a result of folate deficiency: implications for colonic carcinogenesis. Gastroenterology 1996; 110: A503.

63 Maskens AP, Dujardin-Loits R-M. Experimental adenomas and carcinomas of the large intestine behave as distinct entities: most carcinomas arise de novo in flat mucosa. Cancer 1981; 47: 81-9.

64 Nauss KM, Locniskar M, Newberne PM. Effect of alterations in the quality and quantity of dietary fat on 1,2 -dimethylhydrazine-induced colon tumorigenesis in rats. Cancer Res 1983; 43: 4083-90.

65 Nauss KM, Locniskar M, Pavlina T, Newsberne PM. Morphology and distribution of 1,2-dimethylhydrazine dihydrochloride-induced colon tumors and their dihydrochloride-induced colon tumors and their
relationship to gut-associated lymphoid tissue in the rat. relationship to gut-associated lymph
f Natl Cancer Inst 1984; 73: 915-24.

66 Rogers AE, Herndon BJ, Newberne PM. Induction by dimethylhydrazine of intestinal carcinoma in normal rats and rats fed high or low levels of vitamin A. Cancer Res 1973; 33: 1003-9.

67 Barkla DH, Tutton PJM. Surface changes in the descending colon of rats treated with dimethylhydrazine. Cancer Res 1977; 37: 262-71.

68 Decaens C, Gautier R, Daher N, Bara J, Burtin P. Induction of rat intestinal carcinogenesis with single doses, low and high repeated doses of 1,2-dimethylhydrazine. Carcino-
genesis 1989; 10: 69-72.

69 Reddy BS, Watanabe K, Weisburger JH. Effect of high-fat diet on colon carcinogenesis in F344 rats treated with 1,2-dimethylhydrazine, methylazoxymethanol ace

70 Shamsuddin AKM, Trump BF. Colon epithelium. II. In vivo studies of colon carcinogenesis. Light microscopic, histochemical, and ultrastructural studies of histogenesis of azoxymethane-induced colon carcinomas in Fischer 344 rats. $\mathcal{F}$ Natl Cancer Inst 1981; 66: 